\title{
hsa_circRNA_100873 upregulation is associated with increased lymphatic metastasis of esophageal squamous cell carcinoma
}

\author{
BIN ZHENG ${ }^{*}$, ZHIGANG WU* ${ }^{*}$, SONGTAO XUE* ${ }^{*}$ HAO CHEN, SHULIANG ZHANG, \\ TAIDUI ZENG, GUOBING XU, WEIDONG WU, WEI ZHENG and CHUN CHEN
}

\author{
Thoracic Department, Fujian Medical University Union Hospital, Fuzhou, Fujian 350001, P.R. China
}

Received August 21, 2018; Accepted September 6, 2019

DOI: $10.3892 / \mathrm{ol} .2019 .11003$

\begin{abstract}
Circular RNAs (circRNAs) are a type of endogenous non-coding RNA with multiple binding sites that specifically bind to microRNAs (miRNAs) and serve an important role in cellular regulatory networks. Patients exhibit varying levels of lymphatic metastasis in a clinical setting. The present study investigated the association between circRNAs and lymphatic metastasis in esophageal squamous cell carcinoma (ESCC). The tissue samples were divided into three groups, including early tumor stage associated with advanced nodal stage (T1 group), advanced tumor stage associated with early nodal stage (T2 group) and healthy esophageal epithelial tissues as the control group (C group). Gene chip analysis identified circRNAs, and those with possible regulatory functions were validated by reverse transcription-quantitative polymerase chain reaction analysis (RT-qPCR). circRNAs containing miRNA response element (MRE) sequences were obtained, and circRNA/miRNA prediction software was used to predict miRNAs that may interact with circRNA. A total of 12,275 circRNAs were detected, including 861 with statistically significant differences. A comparison between the T1 and C groups identified 152 upregulated circRNAs and 431 downregulated ones, while a comparison between the $\mathrm{T} 2$ and C groups identified 187 upregulated and 481 downregulated circRNAs. A T1/T2 group comparison revealed that four circRNAs were upregulated and seven were downregulated (fold change $>1.5 ; \mathrm{P}<0.05$ ). The RT-qPCR data and gene chip analysis consistently identified hsa_circRNA_100873 as differentially expressed among the examined groups. A total of five potential MREs and complementary sequences were selected for hsa_circRNA_100873. The results of the
\end{abstract}

Correspondence to: Dr Chun Chen, Thoracic Department, Fujian Medical University Union Hospital, 29, Xinquan Road, Fuzhou, Fujian 350001, P.R. China

E-mail: lacustrian@163.com

*Contributed equally

Key words: lymphatic metastasis, esophageal squamous cell carcinoma, circular RNA, hsa_circRNA_100873, expression present study indicated that multiple differentially expressed circRNAs are involved in the pathogenesis of ESCC, and that upregulation of hsa_circRNA_100873 may be associated with increased lymphatic metastases in ESCC.

\section{Introduction}

Esophageal squamous cell carcinoma (ESCC), one of the most common and deadly malignancies, is the eighth most common cancer and the sixth most frequent cause of cancer-associated mortality worldwide (1). The incidence of ESCC in China is higher when compared with western populations, with 477,900 cases reported and the incidence rate accounted for $11.1 \%$ of all malignant tumors. The estimated number of mortalities was 375,000 , accounting for $13.3 \%$ of cancer-associated mortalities in China in 2015 (2,3). Because early symptoms of ESGG are not typical, numerous patients are at an advanced stage at the time of diagnosis, and the majority of patients succumb to the disease due to recurrence or metastasis $(4,5)$. This is corroborated by the 5 -year survival rate, which was only $10-25 \%$ in China in $2015(4,5)$. The precise molecular mechanisms underlying the occurrence, development and lymphatic metastasis of ESCC remain to be fully elucidated. In addition, there are few effective diagnostic markers and therapeutic targets for patients with ESCC, which account in part for its poor prognosis. Therefore, there is an urgent need for the identification of biomarkers or therapeutic targets to improve the clinical outcome of ESCC.

Circular RNAs (circRNAs) are endogenous non-coding RNAs that regulate transcriptional and post-transcriptional gene expression, similar to long non-coding RNAs (lncRNAs) (6). However, unlike lncRNAs, the unique circular structure of circRNAs, without 5' to 3' polarity and without a polyadenylated tail, can prevent degradation by RNA exo-enzymes, which ensures good stability (7). The circRNA loop contains multiple microRNA (miRNA) binding sites. The miRNA binds specifically to corresponding miRNA response elements (MREs) on the circRNA according to the principles of Watson-Crick base pairing, acting as an intracellular miRNA sponge (8). This reduces the extent of miRNA binding to the original target gene, thereby increasing gene expression $(6,8)$. The sponge function of circRNA has been identified in numerous types of cancer, including, lung $(9,10)$, liver $(11,12)$, bladder $(13)$, pancreatic cancer $(14,15)$ and skin 
cancer (16). However, only a few studies have demonstrated the differential expression of circRNAs between ESCC and healthy tissues $(17,18)$, and the specific molecular mechanisms governing this process remain unclear.

In a clinical setting, we found that patients present with varying levels of lymphatic metastasis; even those with early tumor invasion may exhibit advanced lymphatic metastases. The present study investigated the expression of circRNAs and the associations with lymphatic metastasis in ESCC. A circRNA microarray analysis was performed to identify variations in the expression of circRNAs in a range of cancerous tissues with different levels of lymphatic metastases. The present study also investigated potential capabilities through predicting the interactions of circRNA/miRNAs.

\section{Materials and methods}

Patients and samples. A total of six patients aged from 50 to 74 years (5 men and 1 woman) with esophageal cancer and three healthy volunteers aged from 45 to 64 years (1 men and 2 women) who attended Fujian Medical University Union Hospital (Fuzhou, China) between May 2016 and January 2017 were included in the present study. The clinical information of these individuals is presented in Table I. The 6 patients underwent minimally invasive esophagectomy and lymph node dissection, and tumor pathological staging was determined according to the American Joint Committee on tumor-node-metastasis staging criteria (7th edition) (19). Healthy esophageal epithelial tissue was obtained during an esophagoscopy from the 3 healthy volunteers with no recorded tumor complications. The subjects were divided into three groups: Early tumor stage associated with advanced nodal stage (T1N2-3M0; T1 group; $n=3$ ), advanced tumor stage associated with early nodal stage (T3NOM0; T2 group; n=3), and the healthy volunteers as the control group ( $\mathrm{C}$ group; $\mathrm{n}=3$ ).

All tissues samples were snap-frozen in liquid nitrogen immediately following resection, and were then transferred to the Institute of Cardiothoracic Surgery of Fujian Medical University Union Hospital (Fuzhou, China) and cryopreserved at $-80^{\circ} \mathrm{C}$ until use. Total RNA was extracted from the nine specimens with TRIzol ${ }^{\circledR}$ reagent (Invitrogen; Thermo Fisher Scientific, Inc.), according to the manufacturer's protocol. Transformant $1.5 \%$ agarose gel electrophoresis was used to assess RNA integrity and remove genomic DNA contamination. The purity and concentration of total RNA were determined using a NanoDrop ND-1000 spectrophotometer (NanoDrop Technologies; Thermo Fisher Scientific, Inc.). All individuals provided written informed consent prior to inclusion in the study. The study protocol was approved by the Ethics Committee on Human Research of Fujian Medical University (Fuzhou, China).

RNA labeling and hybridization. RNA marker and array hybridization was performed using the Arraystar Super RNA Tag kit (Arraystar Inc.), according to the manufacturer's protocol. Total RNA was treated with RNase R and incubated at $37^{\circ} \mathrm{C}$ for $1 \mathrm{~h}$ (Epicentre; Illumina, Inc.) according to the manufacturer's protocol to remove linear RNA and enrich circRNA. Following the protocol of the Arraystar Super RNA Tag kit, enriched circRNAs were amplified and transcribed
Table I. Clinical characteristics of patients with ESCC and healthy volunteers subjected to circRNA expression profile chip assay.

\begin{tabular}{lccll}
\hline Sample no. & Group & Age, years & Sex & TNM stage \\
\hline 1 & T1 & 71 & Male & T1N2M0 \\
2 & T1 & 74 & Male & T1N2M0 \\
3 & T1 & 50 & Male & T1N3M0 \\
4 & T2 & 65 & Male & T3N0M0 \\
5 & T2 & 65 & Male & T3N0M0 \\
6 & T2 & 68 & Female & T3N0M0 \\
7 & C & 64 & Female & N/A \\
8 & C & 45 & Female & N/A \\
9 & C & 52 & Male & N/A \\
\hline
\end{tabular}

ESCC, esophageal squamous cell carcinoma; circRNA, circular RNA; T1, early tumor stage associated with advanced nodal stage; T2, advanced tumor stage associated with early nodal stage; C, healthy esophageal epithelial tissues; N/A, not applicable; TNM, tumor-node-metastasis.

into fluorescent circRNA utilizing a random priming method. The labelled circRNAs (pmol Cy3/ $\mu$ g cRNA) were purified using the TargetAmp 1-Round RNA Amplification kit 103 (Epicentre; Illumina, Inc.) and the concentration and radioactivity of the labeled circRNA were determined using a NanoDrop ND-1000 spectrophotometer. Subsequently, to cleave circRNA, $5 \mu 1$ 10x blocker and $1 \mu 125 \mathrm{x}$ lysis buffer (Arraystar Super RNA Tag kit; Arraystar) were added per $1 \mu \mathrm{g}$ of sample, and the mixture was heated at $60^{\circ} \mathrm{C}$ for $30 \mathrm{~min}$ until the labeled circRNA became fragmented. Next, $25 \mu 12 \mathrm{x}$ hybridization buffer (Arraystar Super RNA Tag kit; Arraystar) was added to dilute the labeled circRNA samples. Finally, 50 ul labeled circRNA hybridization solution was injected onto the microarray slide and hybridized onto the Arraystar Human Circular RNA Microarray (8_15k; Arraystar, Inc.). After incubation in a $65^{\circ} \mathrm{C}$ Agilent hybridization oven (SureHyb Microarray Hybridization Chamber; Agilent Technologies, Inc.) for $17 \mathrm{~h}$, the hybrid arrays were washed twice with wash buffer at room temperature for 5-10 sec and fixed in the fixation buffer at $25^{\circ} \mathrm{C}$ for $10 \mathrm{~min}$, then scanned by a GenePix 4000B Microarray Scanner (Roche Diagnostics) to generate expression differences in the circRNA expression profile chip.

Microarray data analysis. Data generated from the circRNA microarray underwent data summarization, quantile normalization and quality control using GeneSpring software version 12.0 (Agilent Technologies, Inc.). circRNA for which at least three out of six samples had flags in 'Present' or 'Marginal' ('All Targets Value') were selected for further data analysis. To compare differences in circRNA expression profiles among different groups, the fold-change was calculated between the groups for each circRNA. When comparing the three groups for profile differences, the fold-change for each circRNA was computed, and one-way ANOVA followed by Tukey's post-hoc test were used to analyze statistical differences among three groups (T1, T2 and C) using SPSS version 24 software (IBM 
Table II. Primers used for RT-qPCR analysis of circRNA levels.

Primer sequence (5'-3')

\begin{tabular}{|c|c|c|c|c|}
\hline \multirow[b]{2}{*}{ Gene name } & \multicolumn{2}{|c|}{ Primer sequence $\left(5^{\prime}-3^{\prime}\right)$} & \multirow{2}{*}{$\begin{array}{c}\text { Annealing } \\
\text { temperature, }{ }^{\circ} \mathrm{C}\end{array}$} & \multirow{2}{*}{$\begin{array}{l}\text { Length } \\
\text { bp }\end{array}$} \\
\hline & Forward & Reverse & & \\
\hline$\beta$-actin & GTGGCCGAGGACTTTGATTG & CCTGTAACAACGCATCTCATATT & 60 & 73 \\
\hline hsa_circRNA_402458 & GCACAGTCAGCCAGCCTAATC & TTTTCTCGCACATCCGTTTG & 60 & 125 \\
\hline hsa_circRNA_067567 & GGACTAGGCCCCAATTTAGTG & TCGTGTTTTTACAACTTCCAGTG & 60 & 96 \\
\hline hsa_circRNA_100873 & TGGCCATCCAGGAGATCAT & GGGGAGGTTTCACACTTTATG & 60 & 122 \\
\hline hsa_circRNA_002554 & TGCCAGTTAACAAATAAAATGGA & CAGACTCCTGATGGACCACAAT & 60 & 96 \\
\hline
\end{tabular}

RT-qPCR, reverse transcription-quantitative polymerase chain reaction analysis; circRNA, circular RNA; bp, base pair.

Corp.). $\mathrm{P}<0.05$ and fold-change $>1.5$ indicated a statistically significant difference. The acquired data were filtered and the differentially expressed circRNAs were ranked based on the defined P-value and fold-change parameters.

Reverse transcription-quantitative polymerase chain reaction (RT-qPCR) validation of candidate circRNAs. RT-qPCR was used to verify differentially expressed circRNAs between the T1 and T2 groups. Total RNA was extracted from tissues using the RNA Extraction Kit (Takara Bio, Inc.). cDNA was generated from total RNA using 5X ALL-In-One RT MasterMix (Applied Biosystems) according to the manufacturer's instructions. RT-qPCR reactions were performed as follows: $25^{\circ} \mathrm{C}$ for $10 \mathrm{~min}$, followed by $42^{\circ} \mathrm{C}$ for $50 \mathrm{~min}$ and $85^{\circ} \mathrm{C}$ for $5 \mathrm{~min}$. The relative expression of circRNA was then determined using a sequence-specific oligonucleotide primer designed to generate a 200 bp sequence with the ViiA 7 real-time PCR system (Applied Biosystems; Thermo Fisher Scientific, Inc.) using Hieff ${ }^{\mathrm{TM}}$ qPCR SYBR Green Master Mix (Shanghai Yeasen Biotechnology Co., Ltd.) according to the manufacturer's instructions. The cycling parameters of the PCR reaction were as follows: $95^{\circ} \mathrm{C}$ for $10 \mathrm{~min}$, followed by 40 cycles of $95^{\circ} \mathrm{C}$ for $10 \mathrm{sec}$ and $60^{\circ} \mathrm{C}$ for $60 \mathrm{sec}$. circRNA levels were normalized using $\beta$-actin as an internal control. Data were calculated and run three times using the $2^{-\Delta \Delta \mathrm{Cq}}$ method (20) and quantitative PCR. Primer sequences are presented in Table II. The differences between three groups $(n=3)$ were analyzed by one-way ANOVA followed by Tukey's post-hoc test.

Prediction of circRNA/miRNA interactions. The TargetScan (http://www.targetscan.org/vert_72/) and miRanda (http://www.microrna.org/microrna/home.do) databases were used to facilitate the prediction of circRNA/microRNA interactions, and differentially expressed circRNAs were annotated using circRNA/miRNA interaction information.

\section{Results}

Differentially expressed circRNAs based on microarray analysis. The present study detected a total of 12,275 circRNAs, including 861 circRNAs that were significantly differentially expressed between groups. The distribution of sample intensities was compared using a box plot, and following standardization, the distribution of $\log 2$ ratios for

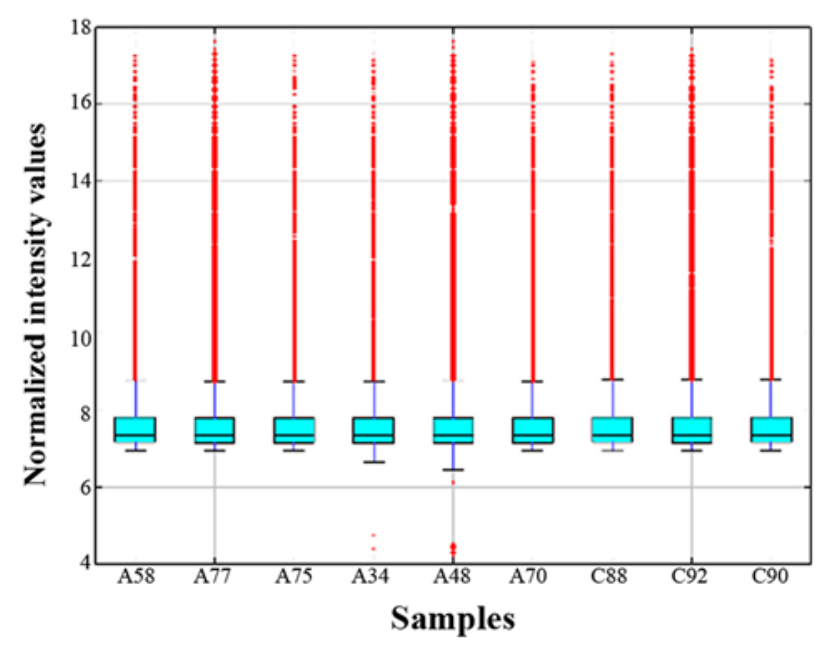

Figure 1. circRNA distribution in all samples. Box plot comparing the distribution of expression values for all samples after normalization. The distribution of circRNAs was similar across all test samples. circRNA, circular RNA.

circRNAs was almost identical in all tested samples (Fig. 1). Scatter plots were used to present differences in the expression of circRNAs among the T1, T2 and C groups. The circRNAs above the top and below the bottom green lines exhibited a fold-change $>1.5$ between the two groups (Fig. 2). Volcano plots were used to visualize the differential expression of circRNAs, with red points representing statistical significance (Fig. 3). The analysis of circRNA expression by hierarchical clustering of heat maps aids hypotheses regarding the association between samples. Hierarchical clustering demonstrated that the circRNA expression profiles were distinguishable in the samples (Fig. 4), and following integration with microarray data differences were detected in the circRNA profiles among the three groups (Figs. 5 and 6).

From the aforementioned analyses, circRNAs were identified that were differentially expressed among the three groups. Of these, there were 152 upregulated circRNAs and 431 downregulated circRNAs in the T1 group compared with the C group, and 187 upregulated circRNAs and 481 downregulated circRNAs in the T2 group compared with the C group (Fig. 4). Additionally, there were four upregulated circRNAs in the $\mathrm{T} 1$ group compared with the T2 group. Two 

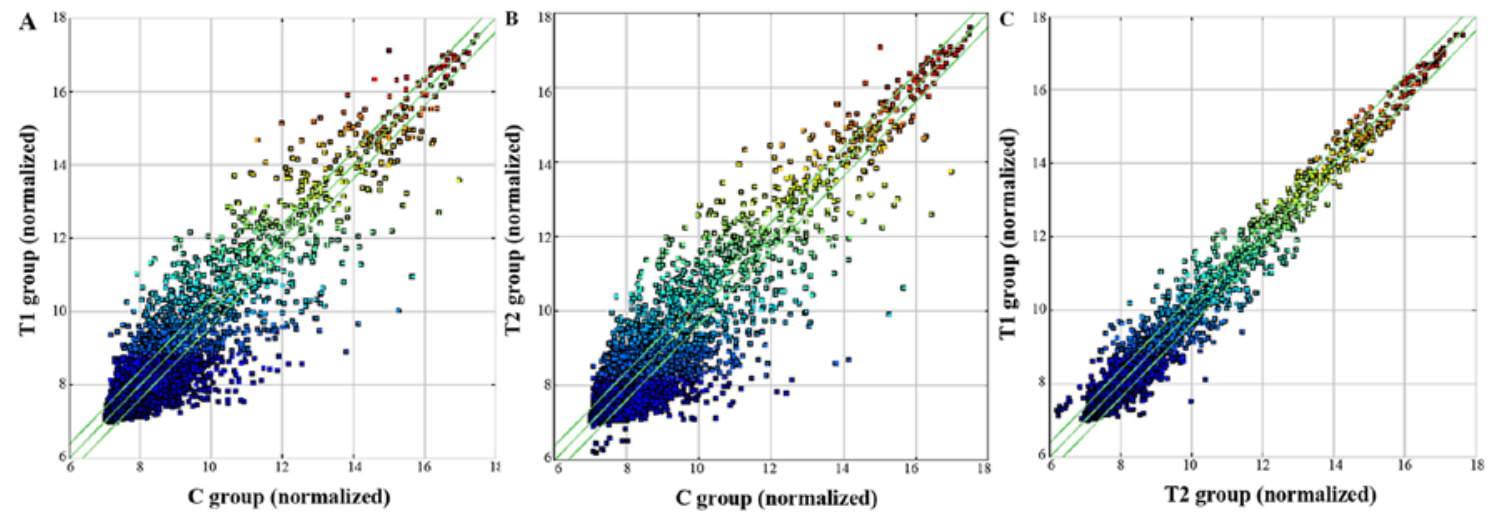

Figure 2. Changes in circRNA expression among groups. Three scatter plots were used to visualize changes in circRNA expression between ESCC tissue and non-tumor tissue groups. (A) The $\mathrm{x}$-axis represents the $\mathrm{C}$ group and the $\mathrm{y}$-axis represents the $\mathrm{T} 1$ group. (B) The $\mathrm{x}$-axis represents the $\mathrm{C}$ group and the $\mathrm{y}$-axis represents the T2 group. (C) The $\mathrm{x}$-axis represents the T2 group and the $\mathrm{y}$-axis represents the $\mathrm{T} 1$ group. The middle green line indicates no difference between the groups, and the area above the upper green line and below the lower green line indicates a fold-change $>1.5$. circRNA, circular RNA; ESCC, esophageal squamous cell carcinoma; T1, early tumor stage associated with advanced nodal stage; T2, advanced tumor stage associated with early nodal stage; C, healthy esophageal epithelial tissues.
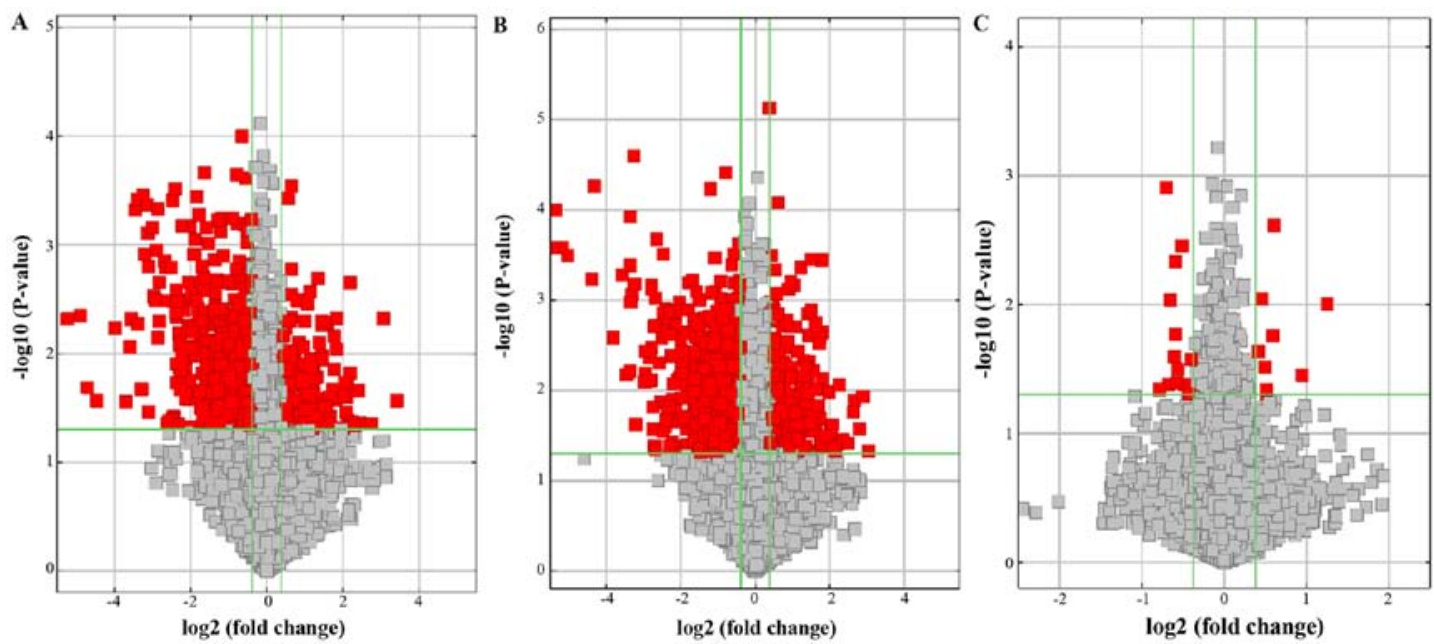

Figure 3. Volcano map to identify circRNAs with statistically significant differences. (A) Comparison of the T1 group and C group. (B) Comparison of the $\mathrm{T} 2$ group and $\mathrm{C}$ group $(\mathrm{C})$ Comparison of the $\mathrm{T} 1$ group and $\mathrm{T} 2$ group. Red dots represent significant differential expression (fold-change $>1.5$ and $\mathrm{P}<0.05$ ). circRNA, circular RNA; T1, early tumor stage associated with advanced nodal stage; T2, advanced tumor stage associated with early nodal stage; C, healthy esophageal epithelial tissues.

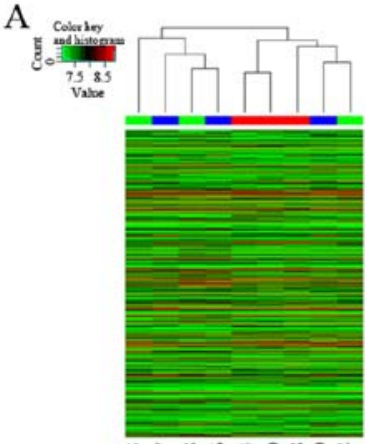

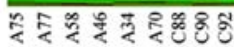

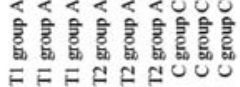

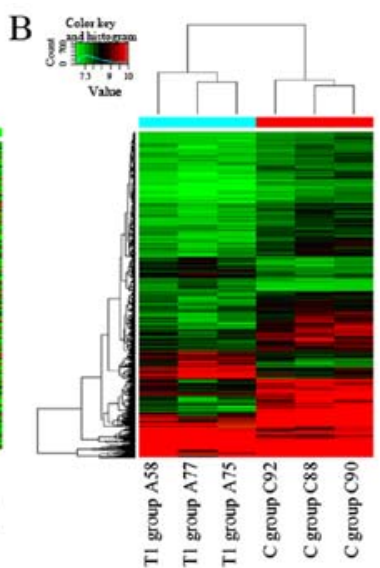

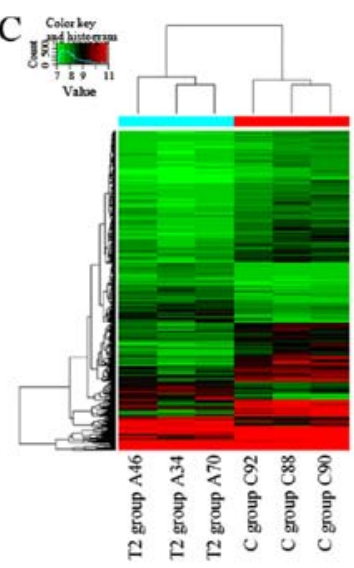

Figure 4. Hierarchical cluster analysis of circRNA. Hierarchical clustering revealed differential circRNA expression in the samples. (A) Hierarchical cluster analysis of circRNAs in the T1, T2 and C groups. (B) Hierarchical cluster analysis of circRNAs in the T1 and C groups. (C) Hierarchical cluster analysis of circRNAs in the T2 and C groups. (D) Hierarchical cluster analysis of circRNAs in the T1 and T2 groups. Each column represents the expression profile of a sample. Red indicates a higher expression level, and green indicates a lower expression level. circRNA, circular RNA; T1, early tumor stage associated with advanced nodal stage; $\mathrm{T} 2$, advanced tumor stage associated with early nodal stage; $\mathrm{C}$, healthy esophageal epithelial tissues. 

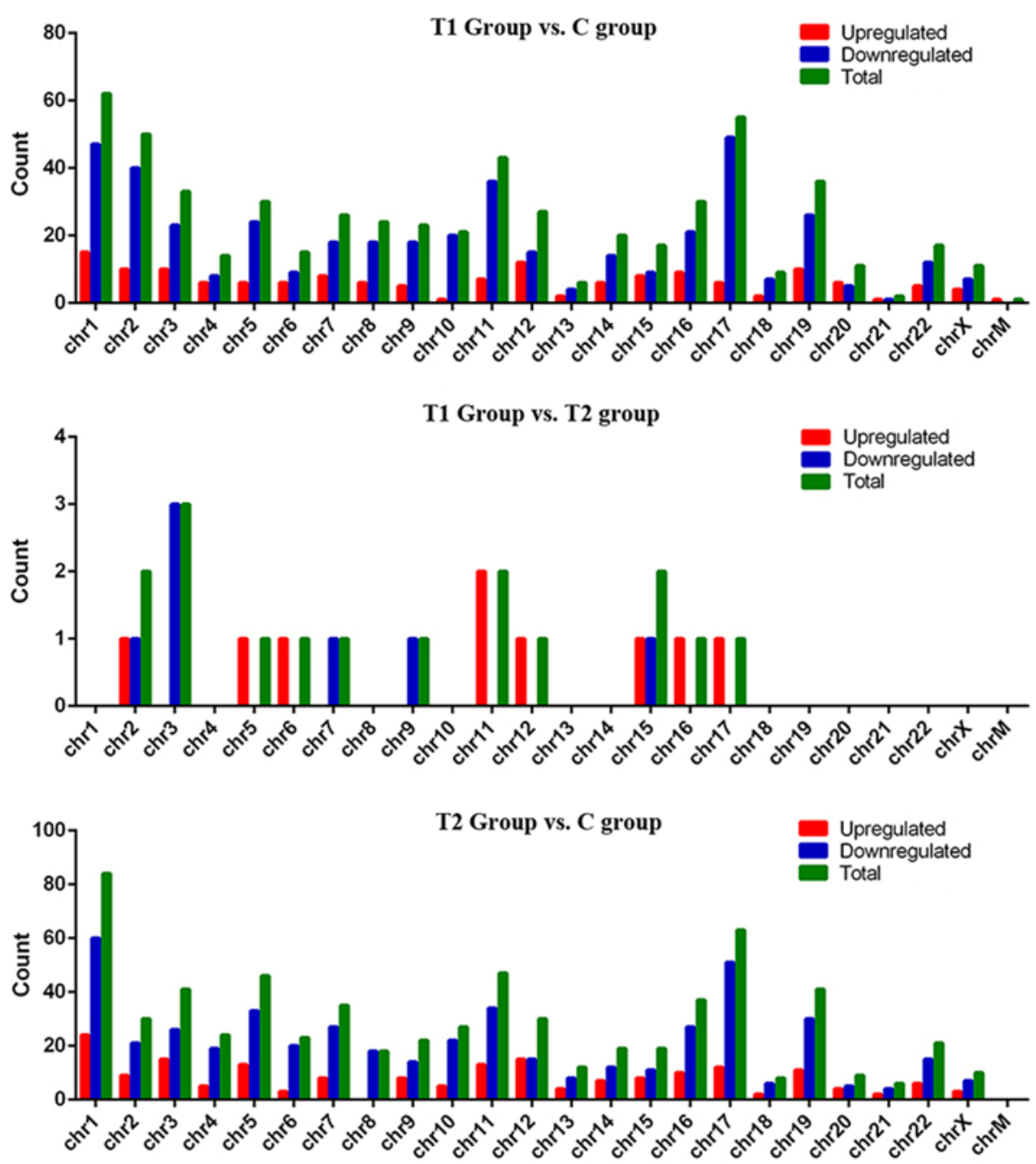

Figure 5. Chromosomal distribution of the differentially expressed circRNAs. circRNA, circular RNA; T1, early tumor stage associated with advanced nodal stage; T2, advanced tumor stage associated with early nodal stage; C, healthy esophageal epithelial tissues.
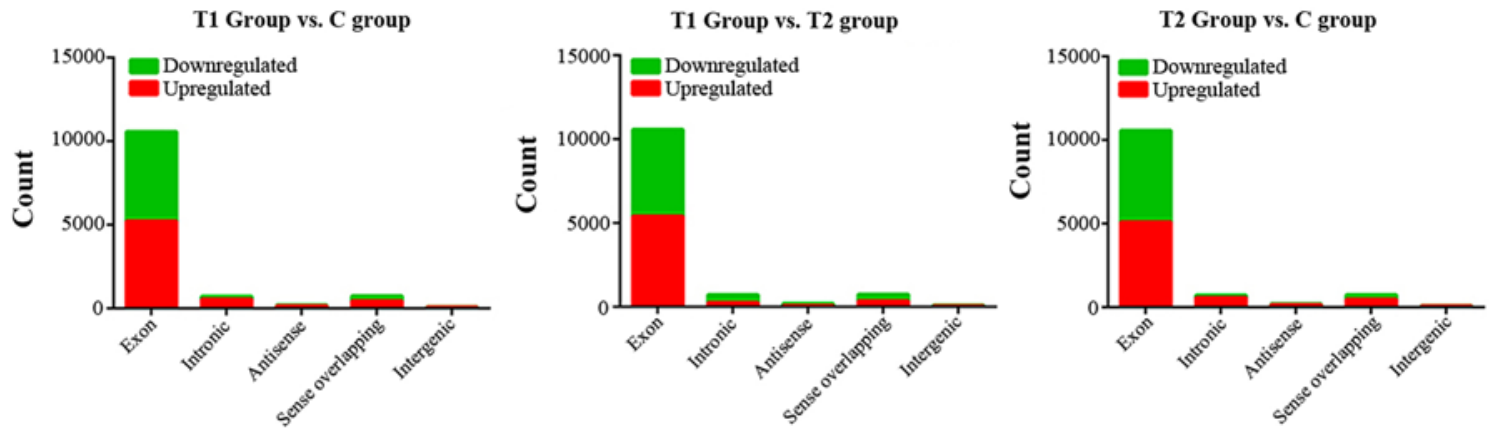

Figure 6. Classification of the differentially expressed circRNAs. circRNA, circular RNA; T1, early tumor stage associated with advanced nodal stage; T2, advanced tumor stage associated with early nodal stage; $C$, healthy esophageal epithelial tissues.

of these (circRNA_402458 and circRNA_100873) were also differentially expressed in the $\mathrm{T} 1$ group or $\mathrm{T} 2$ group compared with the $\mathrm{C}$ group. Seven circRNAs in the T1 group were downregulated compared with the $\mathrm{T} 2$ group, of which two (circRNA_002554 and circRNA_067567) were also differentially expressed in the $\mathrm{T} 1$ group or $\mathrm{T} 2$ group compared with the $\mathrm{C}$ group.
Validation of selected circRNAs using RT-qPCR. The present study selected the following circRNAs: circRNA_402,458, circRNA_100873, circRNA_002554 and circRNA_067567, which were differentially expressed in the $\mathrm{T} 1$ group compared with the T2 group and in the T1/T2 group compared with the $\mathrm{C}$ group, to verify the three groups of samples by RT-qPCR (Fig. 7). hsa_circRNA_100873 (Table III) demonstrated an 

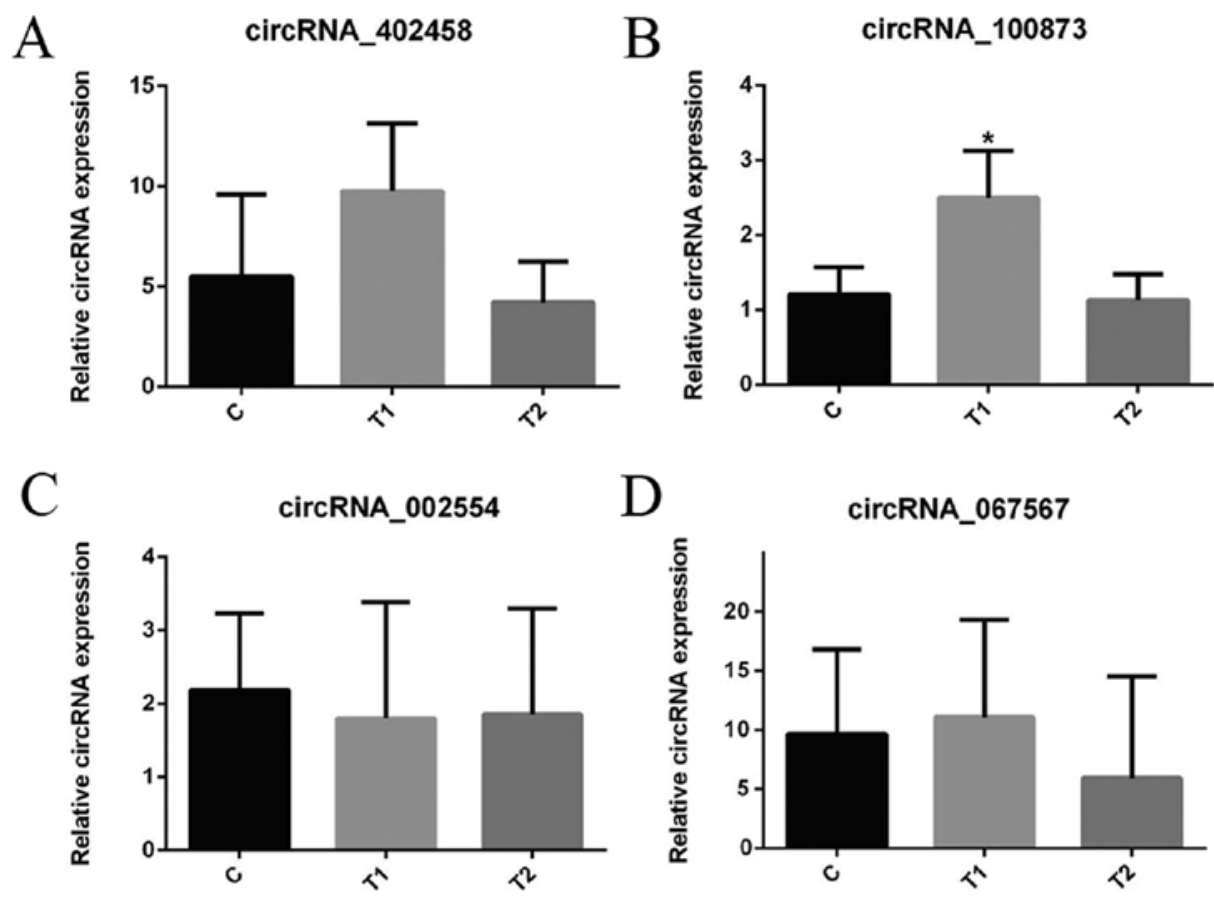

Figure 7. circRNA expression in each group as assessed by RT-qPCR. Expression of (A) circRNA_402458 (B) circRNA_100873 (C) circRNA_002554 and (D) circRNA_067567 in the three groups. There was a significant difference between the T1 group and healthy tissues, and between the T1 group and T2 group. "P<0.05 vs. C and T2). circRNA, circular RNA; RT-qPCR, reverse transcription-quantitative polymerase chain reaction; T1, early tumor stage associated with advanced nodal stage. T2, advanced tumor stage associated with early nodal stage. $\mathrm{C}$, healthy esophageal epithelial tissues.

expression pattern consistent with microarray data analysis for the four circRNAs. There was a significant difference in hsa_circRNA_100873 expression between tissues in the T1 group and T2/C groups (Table IV).

Prediction of circRNA/miRNA interactions. According to the competing endogenous RNA hypothesis and previous reports, circRNAs can interact with specific miRNAs through base complementation via MREs, in a function known as the miRNA sponge (6,21-23). Therefore, the present study used an in-house miRNA target prediction software from Arraystar to predict miRNAs that may bind to the selected circRNAs. The top five miRNAs predicted to pair with circRNA_100873 were identified as hsa-miR-1236-3p, hsa-miR-3064-5p, hsa-miR-6504-5p, hsa-miR-943 and hsa-miR-522-3p (Fig. 8).

\section{Discussion}

circRNAs exist in the cytoplasm of eukaryotic cells and their contents are very similar to classical linear RNAs (7). However, their circular structure is more stable than linear structures; therefore, the circular configuration is often adopted in the cell $(7,8,24)$. Several studies have demonstrated that circRNAs exhibit tissue specificity during normal tissue differentiation and development, and have multiple regulatory functions during the generation and progression of disease, including the regulation of Wnt signaling pathways and epithelial-mesenchymal transition $(6,8,17,25)$. One of the best known is the sponge function of circRNA discovered by Hansen et al (6). This involves circRNAs binding to specific miRNAs, which reduces the miRNA content and weakens inhibition of the original target mRNA, thus changing the expression of the corresponding protein (6).
Numerous examples of circRNA sponges have been identified, including ciRS-7 (6), sex determination region Y (SRY) (6,21), hsacirc001569 (22), circPVT1 (23), circTCF25 (26) and cir-E3 ubiquitin ligase (ITCH; 17). ciRS-7 has $>70$ valid MREs, including miR-7 $(6,8,27,28)$, while SRY was demonstrated to be the sponge of miR-138 $(6,21)$. These circRNAs participate in the development of specific tumorigenesis by binding to specific miRNAs. Thus, circRNAs are promising targets for the diagnosis and treatment of disease.

Few circRNAs have been reported in the field of ESCC. Li et al (17) reported that cir-ITCH is expressed at low levels in ESCC and promotes the ubiquitination and degradation of phosphorylated Dv12 by increasing ITCH expression through the sponge action of miR-7, miR-17 and miR-214. In turn, cir-ITCH inhibits the Wnt/ $\beta$-catenin pathway. Xia et al (29) reported a significant increase in the novel circRNA hsa_circ_0067934 in the cytoplasm of esophageal cancer, and revealed that small interfering RNA-mediated silencing of hsa_circ_0067934 inhibits the invasion and migration of ESCC cells in vitro and blocks cell cycle progression, indicating its potential as a novel biomarker and therapeutic target. Su et al (18) performed a circRNA microarray and bioinformatics analysis on the expression of circRNA in radioresistant and non-radioresistant ESCC cells. They identified RNAs with significantly different expression and proposed that $>400$ target genes were enriched in the Wnt signaling pathway. Of these, circRNA_001059 and circRNA_000167 were the two largest nodes in the circRNA/miRNA co-expression network. Sang et al (30) reported significant upregulation of ciRS-7 in ESCC, which was associated with significant increases in the proliferation, migration and invasion of ESCC cells. High-throughput experiments identified 19 miR-876-5p binding sites in ciRS-7, 
Table III. Expression of has_circRNA_10087.

\begin{tabular}{lcccccccc}
\hline circRNA & P-value & FDR & $\begin{array}{c}\text { Fold } \\
\text { change }\end{array}$ & Regulation & Chrom & $\begin{array}{c}\text { circRNA } \\
\text { type }\end{array}$ & $\begin{array}{c}\text { Sest } \\
\text { Strand }\end{array}$ & $\begin{array}{c}\text { Gene } \\
\text { transcript } \\
\text { symbol }\end{array}$ \\
\hline hsa_circRNA_100873 & 0.035 & 0.999915 & 1.93 & Up & chr11 & Exonic & + & NM_003626 PPFIA1 \\
\hline
\end{tabular}

PPFI1A, PRPRF interacting protein $\alpha$ 1; FDR, false discovery rate; circRNA, circular RNA.

Table IV. Four circRNAs demonstrate differential expression in the three groups.

\begin{tabular}{lcccc}
\hline Comparison & hsa_circRNA_402458 & hsa_circRNA_100873 & hsa_circRNA_002554 & hsa_circRNA_067567 \\
\hline T1 vs. C & 1.790 & 2.120 & 0.930 & 1.030 \\
P-value & 0.382 & 0.037 & 0.989 & 0.996 \\
T2 vs. C & 0.760 & 0.940 & 0.960 & 0.880 \\
P-value & 0.903 & 0.981 & 0.996 & 0.919 \\
T1 vs. T2 & 2.350 & 2.270 & 0.970 & 1.170 \\
P-value & 0.227 & 0.030 & 0.998 & 0.882 \\
\hline
\end{tabular}

circular RNA; T1, early tumor stage associated with advanced nodal stage; T2, advanced tumor stage associated with early nodal stage; C, healthy esophageal epithelial tissues.

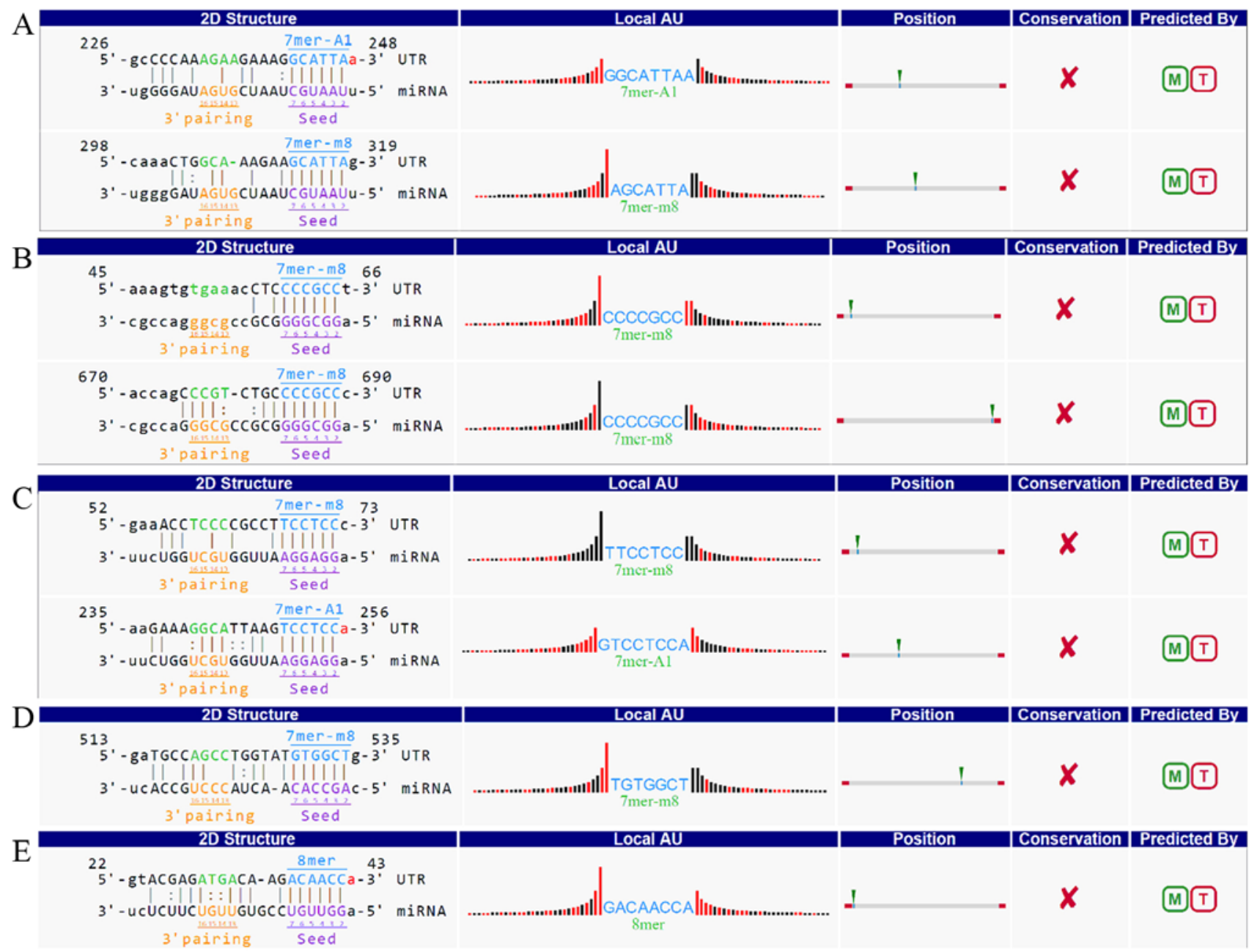

Figure 8. Top five miRNAs predicted to bind with circRNA_100873. (A) hsa-miR-1236-3p; (B) hsa-miR-3064-5p; (C) hsa-miR-6504-5p; (D) hsa-miR-943; and (E) hsa-miR-522-3p. miRNA, microRNA; AU, miRNA binding sites; M, miRanda database; T, TargetScan database. 
suggesting it is a sponge for miR-876-5p. In addition, upregulating ciRS-7 enhanced the growth and metastasis of ESCC tumors by targeting the miR-876-5p/MAGE-A family axis. This was further confirmed in animal studies (30). Thus, circRNAs serve an important role in the development of ESCC.

We have previously detected differences, albeit insignificant, in circRNA expression between different $\mathrm{N}$ stage tumor tissues with the same $\mathrm{T}$ stage. Therefore, the present study examined factors that influence metastasis and tumor development. T1 was defined as a group of early tumor stage associated with advanced nodal stage with extremely high metastatic ability, and a T2 group of advanced tumor stage associated with early nodal stage with relatively lower metastatic ability. The present study investigated circRNA expression profiles in ESCC by comparing low-invasive high-lymphatic metastasis, high-invasive low-lymphatic metastasis and healthy tissue to identify differential hsa_circRNA_100873 expression in the T1/C and T1/T2 groups, as verified by RT-qPCR. The circular RNA differential expression was not only between tumor tissue and healthy tissue, but also between high-lymphatic metastasis tissue and low-lymphatic metastasis tissue, which may suggest a key function in tumor metastasis. Further prediction of circRNA/miRNA interactions identified five potential binding sites in has_circRNA_100873: hsa-miR-155-5p,hsa-miR-663a, hsa-miR-766-5p, hsa-miR-449b-3p and hsa-miR-494-5p.

Shi et al $(31,32)$ observed that hsa-miR-663a directly targets phosphatidylinositol-4,5-bisphosphate 3-kinase catalytic subunit $\delta$ in glioblastoma and downregulated three critical downstream effectors of phosphorylated AKT and PIK3D, including cyclin D1, matrix metallopeptidase (MMP)2 and MMP7, which inhibit tumors. They also identified that hsa-miR-663a functioned as a tumor suppressor by downregulating $\mathrm{C}-\mathrm{X}-\mathrm{C}$ chemokine receptor type 4. Zang et al (33) demonstrated that hsa-miR-663a and eEF1A2 were negatively correlated with each other in pancreatic cancer, and that hsa-miR-663a inhibited the invasion and metastasis of pancreatic cancer cells in vitro and in vivo by directly targeting eEF1A2. Ma et al (34) reported that epithelial membrane protein 3 (EMP3) is a target gene of hsa-miR-663a in gallbladder carcinoma, and that hsa-miR-663a overexpression downregulates EMP3 and activates mitogen-activated protein kinase/extracellular signal-regulated kinase signaling, which directly affects cell invasion and metastasis. Additionally, in liver cancer and non-small cell lung cancer $(35,36)$, hsa-miR-663a inhibits tumor cell proliferation and metastasis by targeting high mobility group AT-hook 2 and JunD, respectively. The role of hsa-miR-663a in inhibiting tumor growth has also been reported in gastric cancer (37), papillary thyroid carcinoma (38) and chronic myeloid leukemia (39). Therefore, the present study hypothesizes that hsa-miR-663a is likely to serve an important role in the invasion and metastasis of esophageal cancer as a sponge of hsa_circRNA_100873. hsa_circRNA_100873 is an exon-derived RNA that may downregulate miRNA hsa-miR-663a through direct binding, thereby regulating metastasis and invasion in ESCC cells.

In summary, the present study examined the expression patterns of circRNAs between a low-invasive high-lymphatic metastasis group, a high-invasive low-lymphatic metastasis group and a healthy esophageal epithelial tissue group. It was observed that, compared with healthy tissues, hsa circRNA_100873 was upregulated in cancer tissues and differentially expressed in low-invasive high-lymphatic metastasis and high-invasive low-lymphatic metastasis ESCC, confirming its regulatory role in the invasion and metastasis of ESCC cells. However, the present study has certain limitations. Future experiments should verify the circRNA expression levels in a higher number of pathological samples and conduct a series of associated phenotypic and mechanism experiments. This circRNA, hsa_circRNA_100873, may be a potential novel target for the diagnosis and treatment of ESCC, although its specific molecular biological mechanism requires further research.

\section{Acknowledgements}

Not applicable.

\section{Funding}

The present study was financially supported by the Fujian Provincial Key Project (grant no. 2014Y0024), the Fujian Provincial Joint Research Project of Health Care and Education (grant no. WKJ2016-2-09) and the Science and Technology Innovation Joint Fund Project (grant no. 2018Y9058).

\section{Availability of data and materials}

The datasets used and/or analyzed during the present study are available from the corresponding author on reasonable request.

\section{Authors' contributions}

CC, BZ and WZ undertook study conception and design, and provided administrative support. BZ, ZW and SX provided the study materials. ZW, SX and WW undertook the collection and assembly of data. HC, SZ, TZ and GX performed data analysis and interpretation. All authors undertook manuscript writing and gave final approval of manuscript to be published.

\section{Ethics approval and consent to participate}

All patients provided written informed consent for participation, and the study protocol was approved by the Ethics Committee on Human Research of Fujian Medical University (Fuzhou, China).

\section{Patient consent for publication}

Not applicable.

\section{Competing interests}

The authors declare that they have no competing interests.

\section{References}

1. Bray F, Jemal A, Grey N, Ferlay J and Forman D: Global cancer transitions according to the human development index (2008-2030): A population-based study. Lancet Oncol 13: 790-801, 2012. 
2. Chen W, Zheng R, Baade PD, Zhang S, Zeng H, Bray F, Jemal A Yu XQ and He J: Cancer statistics in china, 2015. CA Cancer J Clin 66: 115-132, 2016.

3. Abnet CC, Arnold M and Wei WQ: Epidemiology of esophageal squamous cell carcinoma. Gastroenterology 154: 360-373, 2018.

4. Wang W, Wei C, Li P, Wang L, Li W, Chen K, Zhang J, Zhang W and Jiang G: Integrative analysis of mRNA and lncRNA profiles identified pathogenetic lncRNAs in esophageal squamous cell carcinoma. Gene 661: 169-175, 2018.

5. Mariette C, Balon JM, Piessen G, Fabre S, Van Seuningen I and Triboulet JP: Pattern of recurrence following complete resection of esophageal carcinoma and factors predictive of recurrent disease. Cancer 97: 1616-1623, 2003.

6. Hansen TB, Jensen TI, Clausen BH, Bramsen JB, Finsen B Damgaard CK and Kjems J: Natural RNA circles function as efficient microRNA sponges. Nature 495: 384-388, 2013.

7. Nigro JM, Cho KR, Fearon ER, Kern SE, Ruppert JM, Oliner JD, Kinzler KW and Vogelstein B: Scrambled exons. Cell 64: 607-613, 1991

8. Memczak S, Jens M, Elefsinioti A, Torti F, Krueger J, Rybak A, Maier L, Mackowiak SD, Gregersen LH, Munschauer M, et al Circular RNAs are a large class of animal RNAs with regulatory potency. Nature 495: 333-338, 2013

9. Xu J, Shu Y, Xu T, Zhu W, Qiu T, Li J, Zhang M, Xu J, Guo R, $\mathrm{Lu} \mathrm{K}$, et al: Microarray expression profiling and bioinformatics analysis of circular RNA expression in lung squamous cell carcinoma. Am J Transl Res 10: 771-783, 2018.

10. Luo YH, Zhu XZ, Huang KW, Zhang Q, Fan YX, Yan PW and Wen J: Emerging roles of circular RNA hsa_circ_0000064 in the proliferation and metastasis of lung cancer. Biomed Pharmacother 96: 892-898, 2017.

11. Lin Q, Ling YB, Chen JW, Zhou CR, Chen J, Li X and Huang MS Circular RNA circCDK13 suppresses cell proliferation, migration and invasion by modulating the JAK/STAT and PI3K/AKT pathways in liver cancer. Int J Oncol 53: 246-256, 2018.

12. Jiang W, Wen D, Gong L, Wang Y, Liu Z and Yin F: Circular RNA hsa_circ_0000673 promotes hepatocellular carcinoma malignance by decreasing mir-767-3p targeting set. Biochem Biophys Res Commun 500: 211-216, 2018.

13. Yang C, Yuan W, Yang X, Li P, Wang J, Han J, Tao J, Li P, Yang H, Lv Q and Zhang W: Circular RNA circ-ITCH inhibits bladder cancer progression by sponging miR-17/miR-224 and regulating p21, PTEN expression. Mol Cancer 17: 19, 2018.

14. Xu C, Yu Y and Ding F: Microarray analysis of circular RNA expression profiles associated with gemcitabine resistance in pancreatic cancer cells. Oncol Rep 40: 395-404, 2018.

15. Huang WJ, Wang Y, Liu S, Yang J, Guo SX, Wang L, Wang H and Fan YF: Silencing circular RNA hsa_circ_0000977 suppresses pancreatic ductal adenocarcinoma progression by stimulating miR-874-3p and inhibiting PLK1 expression. Cancer Lett 422: 70-80, 2018

16. Sand M, Bechara FG, Gambichler T, Sand D, Bromba M, Hahn SA, Stockfleth E and Hessam S: Circular RNA expression in cutaneous squamous cell carcinoma. J Dermatol Sci 83: $210-218,2016$

17. Li F, Zhang L, Li W, Deng J, Zheng J, An M, Lu J and Zhou Y: Circular RNA itch has inhibitory effect on ESCC by suppressing the Wnt/ $\beta$-catenin pathway. Oncotarget 6: 6001-6013, 2015.

18. Su H, Lin F, Deng X, Shen L, Fang Y, Fei Z, Zhao L, Zhang X, Pan H, Xie D, et al: Profiling and bioinformatics analyses reveal differential circular RNA expression in radioresistant esophageal cancer cells. J Transl Med 14: 225, 2016.

19. Edge SB, Byrd DR, Compton CC, Fritz AG, Greene FL and Trotti A, (eds). AJCC cancer staging manual (7th edition). New York, NY, Springer, 2010

20. Livak KJ and Schmittgen TD: Analysis of relative gene expression data using real-time quantitative PCR and the 2(-Delta Delta C(T)) Method. Methods 25: 402-408, 2001

21. Granados-Riveron JT and Aquino-Jarquin G: Does the linear Sry transcript function as a ceRNA for miR-138? The sense of antisense. Version 2. F1000Res 3: 90, 2014.
22. Xie H, Ren X, Xin S, Lan X, Lu G, Lin Y, Yang S, Zeng Z, Liao W, Ding YQ and Liang L: Emerging roles of circrna_001569 targeting miR-145 in the proliferation and invasion of colorectal cancer. Oncotarget 7: 26680-26691, 2016.

23. Chen J, Li Y, Zheng Q, Bao C, He J, Chen B, Lyu D, Zheng B, $\mathrm{Xu}$ Y, Long Z, et al: Circular RNA profile identifies circPVT1 as a proliferative factor and prognostic marker in gastric cancer. Cancer Lett 388: 208-219, 2017.

24. Zhang XO, Wang HB, Zhang Y, Lu X, Chen LL and Yang L: Complementary sequence-mediated exon circularization. Cell 159: 134-147, 2014.

25. Conn SJ, Pillman KA, Toubia J, Conn VM, Salmanidis M, Phillips CA, Roslan S, Schreiber AW, Gregory PA and Goodall GJ: The RNA binding protein quaking regulates formation of circRNAs. Cell 160: 1125-1134, 2015.

26. Zhong Z, Lv M and Chen J: Screening differential circular RNA expression profiles reveals the regulatory role of circTCF25-miR-103a-3p/miR-107-CDK6 pathway in bladder carcinoma. Sci Rep 6: 30919, 2016

27. Hansen TB, Wiklund ED, Bramsen JB, Villadsen SB, Statham AL, Clark SJ and Kjems J: miRNA-dependent gene silencing involving Ago2-mediated cleavage of a circular antisense RNA. EMBO J 30: 4414-4422, 2011.

28. Zhao Y, Alexandrov PN, Jaber V and Lukiw WJ: Deficiency in the ubiquitin conjugating enzyme UBE2A in Alzheimer's disease (AD) is linked to deficits in a natural circular miRNA-7 sponge (circRNA; ciRS-7). Genes (Basel) 7: pii: E116, 2016.

29. Xia W, Qiu M, Chen R, Wang S, Leng X, Wang J, Xu Y, Hu J, Dong G, Xu PL and Yin R: Circular RNA has_circ_0067934 is upregulated in esophageal squamous cell carcinoma and promoted proliferation. Sci Rep 6: 35576, 2016

30. Sang M, Meng L, Sang Y, Liu S, Ding P, Ju Y, Liu F, Gu L, Lian Y, Li J, et al: Circular RNA cirs-7 accelerates ESCC progression through acting as a miR-876-5p sponge to enhance MAGE-a family expression. Cancer Lett 426: 37-46, 2018.

31. Shi Y, Chen C, Zhang X, Liu Q, Xu JL, Zhang HR, Yao XH, Jiang T, He ZC, Ren Y, et al: Primate-specific miR-663 functions as a tumor suppressor by targeting PIK3CD and predicts the prognosis of human glioblastoma. Clin Cancer Rse 20: 1803-1813, 2014

32. Shi Y, Chen C, Yu SZ, Liu Q, Rao J, Zhang HR, Xiao HL, Fu TW, Long H, He ZC, et al: Mir-663 suppresses oncogenic function of CXCR4 in glioblastoma. Clin Cancer Res 21: 4004-4013, 2015.

33. Zang W, Wang Y, Wang T, Du Y, Chen X, Li M and Zhao G: miR-663 attenuates tumor growth and invasiveness by targeting eEF1A2 in pancreatic cancer. Mol Cancer 14: 37, 2015.

34. Ma Q, Zhang Y, Liang H, Zhang F, Liu F, Chen S, Hu Y, Jiang L, Hao Y, Li M and Liu Y: EMP3, which is regulated by miR-663a, suppresses gallbladder cancer progression via interference with the MAPK/ERK pathway. Cancer Lett 420: 97-108, 2018.

35. Huang W, Li J, Guo X, Zhao Y and Yuan X: miR-663a inhibits hepatocellular carcinoma cell proliferation and invasion by targeting HMGA2. Biomed Pharmacother 81: 431-438, 2016.

36. Zhang Y, Zhou X, Xu X, Zhang M, Wang X, Bai X, Li H, Kan L, Zhou Y, Niu $\mathrm{H}$ and $\mathrm{He} \mathrm{P}$ : Waltonitone induces apoptosis through mir-663-induced Bcl-2 downregulation in non-small cell lung cancer. Tumour Biol 36: 871-876, 2015.

37. Pan J, Hu H, Zhou Z, Sun L, Peng L, Yu L, Sun L, Liu J, Yang Z and Ran Y: Tumor-suppressive mir-663 gene induces mitotic catastrophe growth arrest in human gastric cancer cells. Oncol Rep 24: 105-112, 2010.

38. Wang Z, Zhang H, Zhang P, Dong W and He L: MicroRNA-663 suppresses cell invasion and migration by targeting transforming growth factor beta 1 in papillary thyroid carcinoma. Tumour Biol 37: 7633-7644, 2016.

39. Yang Y, Wang LL, Wang HX, Guo ZK, Gao XF, Cen J, Li YH, Dou LP and Yu L: The epigenetically-regulated miR-663 targets H-Ras in K-562 cells. FEBS J 280: 5109-5117, 2013. 